\title{
Decentralized Spectral Resource Allocation for OFDMA Downlink of Coexisting Macro/Femto Networks using Filled Function Method
}

\author{
L. Benmesbah¹, B.W.K. Ling ${ }^{1}$, Senior Member of IEEE, V. Chandrasekhar ${ }^{2}$, X. Chu' ${ }^{1}$, and M. Dohler ${ }^{3}$ \\ ${ }^{1}$ Department of Electronic Engineering, King's College London, London, United Kingdom \\ ${ }^{2}$ Department of Electrical and Computer Engineering, University of Texas at Austin, Austin, United States of America \\ ${ }^{3}$ Centre Tecnològic de Telecomunicacions de Catalunya, Parc Mediterrani de la Tecnologia, Barcelona, Spain \\ lamia.benmesbah@kcl.ac.uk, wing-kuen.ling@kcl.ac.uk, cvikram@mail.utexas.edu, xiaoli.chu@kcl.ac.uk, and \\ mischa.dohler@cttc.es
}

\begin{abstract}
For an orthogonal frequency division multiple access (OFDMA) downlink of a spectrally coexisting macro and femto network, a resource allocation scheme would aim to maximize the area spectral efficiency (ASE) subject to constraints on the radio resources per transmission interval accessible by each femtocell. An optimal resource allocation scheme for completely decentralized deployments leads however to a nonconvex optimization problem. In this paper, a filled function method is employed to find the global maximum of the optimization problem. Simulation results show that our proposed method is efficient and effective.
\end{abstract}

\section{INTRODUCTION}

Mobile user equipments (UEs) have become increasingly important in our daily lives and mobile operators have been spending a lot of money to meet the growing customer demands. However, most current cellular networks suffer from poor indoor network coverage and cellsite usage saturation. As a consequence, mobile operators face poor customer retain-ability and increasing churn. One cost-effective solution for mobile operators to improve coverage and offload macrocell traffic as well as to reduce churn is the emerging femtocell paradigm. Typically, a large number of femtocell access points (FAPs) [1] are overlaid on macrocells. Each FAP provides high data-rate connections to UEs within a short range using the same radio access technology as the macrocell overlay. As OFDMA has been considered in the downlink for next generation wireless networks [2]-[3], OFDMA based femtocells are widely expected to deliver massive improvements in coverage and capacity [1].

A pertinent challenge for mobile operators is to viably deploy femtocells within coverage of macrocells. As FAPs are likely to be deployed by end users, the total number and locations of active FAPs are a priori unknown to mobile operators. Therefore, interference caused by femtocells cannot be managed using conventional network planning methods, and interference avoidance strategies [4] are preferred over mutual interference suppression strategies for femtocells. Intercell interference in the hierarchical macro/femto network is closely related to the femtocell access policy, which defines the way how a femtocell allows or restricts its usage to users [4]. This paper will thus focus on the closed access femtocell, which serves a group of authorized UEs only, since the closed access policy is preferred by subscribers of the femtocell [5].

OFDMA radio resources are partitioned into resource blocks (RBs) in the time and frequency domains [15]. To mitigate intercell interference, frequency reuse [6]-[8] and intercell coordination [9], [10] schemes have been studied in OFDMA macro networks. A centralized downlink frequency planning across femtocells and macrocells was proposed in [11], but the large number of femtocells significantly complicates the centralized optimization process.

The spectrum allocation policy in [12] avoids cross-tier interference by assigning orthogonal spectrum resources to the macrocell and femtocell tiers and diminishes femtoto-femto interference by allowing each femtocell to access only a random subset of the spectrum resources that are assigned to the femtocell tier. Specifically for each transmission time interval, a macrocell can use all the available RBs, while each femtocell randomly selects a subset of the available RBs for transmissions [12]. As a result, the average number of interfering femtocells in each RB is reduced. The size of each RB subset per transmission interval is determined based on optimizing the throughput per cell and ASE [12]. To optimize such a decentralized resource allocation leads to a nonconvex optimization problem, the global maximum of which is very difficult to find. Therefore, existing works [12]-[14] on decentralized resource allocation for the OFDMA downlink of macro/femto networks reduce to evaluate all possible values of the fraction of radio resources per transmission interval accessible by each femtocell.

In this paper, we employ the filled function method [20]-[25] for solving the global maximum of the optimization problem. Our proposed method only needs to evaluate the stationary points of the cost function, thereby significantly reduces the total number of evaluation points, and the computational complexity for finding the globally optimal solution of the optimization problem is much lower than the that of scheme in [12].

The rest of the paper is organized as follows. The problem formulation and the filled function method are presented in Section II and Section III, respectively. Simulation results are discussed in Section IV. Finally, conclusions are drawn in Section V. 


\section{PROBLEM Formulation}

Consider an OFDMA downlink of a spectrally coexisting macro/femto network. It is assumed that the macro base station (BS) $B_{0}$ is located in the middle of the macrocell and co-channel interference from neighboring macrocell transmissions is ignored, as extending the work to the more general case would be cumbersome [17]. Denote the radius of a hexagonal macrocell as $r_{M}$ and the number of outdoor macro UEs randomly distributed over the macrocell coverage area as $U_{M}$. Closed access femtocells are randomly underlaid to the macrocell and the location of the FAPs is a stationary Poisson point process (SPPP) [16] on $\mathfrak{R}^{2}$. The spatial intensity of FAPs $\lambda_{F}$ is defined as the average number of femtocells per unit area. The radius of each femtocell is $r_{F}$. The number of indoor UEs to be served by each femtocell is $U_{F}$. If the average number of femtocells per macrocell is $N_{F}$, then total number of UEs served by the coexisting macro/femto network is given by $U=U_{M}+N_{F} U_{F}$.

The basic resource unit that is addressable for OFDMA transmissions is an RB. RB is a two dimensional block in the time and frequency domain. In the third generation partnership project (3GPP) standard release 8 [15], an RB has a time duration of $1 \mathrm{~ms}$ and spans either 12 subcarriers with a subcarrier bandwidth of $15 \mathrm{kHz}$ or 24 subcarriers with a subcarrier bandwidth of $7.5 \mathrm{kHz}$. Intracell interference is avoided by maintaining the orthogonality among co-cell UEs in OFDMA networks [12], [18]; that is, maintaining one scheduled UE per RB in each cell. Not to clutter subsequent analysis, it is assumed that a macro BS or an FAP assigns equal transmission power all over RBs for a given transmission interval [12]. Since cellular networks are typically interference limited, thermal noise at the receiver is neglected in this paper.

As in [12], we assume that the macrocell and femtocells are allocated with orthogonal spectrum resources and there is not macro-to-femto or femto-to-macro interference. Therefore, we can focus on the decentralized resource allocation between femtocells only. Let the total number of distinct RBs available to femtocells for each transmission time interval be $F \in Z^{+}$. Each FAP is only allowed to use the available $K \in Z^{+}(K \leq F)$ RBs for downlink transmissions in each transmission time interval. For ease of implementation, the value of $K$ is assumed to be the same for all femtocells. Define the fraction of radio resources per transmission interval accessible by each femtocell as $\rho_{F}$. That is

$$
\rho_{F}=\frac{K}{F} .
$$

Obviously, $0 \leq \rho_{F} \leq 1$. When $\rho_{F}=1$, each femtocell can access all the available RBs.

If each femtocell chooses the $K$ distinct RBs independently and with equal probability, then the probability of a femtocell selecting a given RB for transmission is $\rho_{F}$. This implies that the average number of interfering femtocells in each RB is effectively reduced. Hence, both femto to macro and femto to femto interferences are diminished.

Define the total number of discrete rates that an adaptive modulation is realizing as $L>0$, the Shannon gap of QAM modulations [12] as $\Psi$, the boundaries of the range of signal interference ratio (SIR) as $\Gamma_{l}$ for $l \in\{1, \cdots, L\}$ and the transmission rates as $b_{l}$ for $l \in\{1, \cdots, L\}$. When the received SIR lies in $\left[\Gamma_{l}, \Gamma_{l+1}\right)$ for $l \in\{1, \cdots, L\}$, the BS or FAP decides the transmission rate according to

$$
b_{l}=\log _{2}\left(1+\frac{\Gamma_{l}}{\Psi}\right),(\mathrm{b} / \mathrm{s} / \mathrm{Hz}) .
$$

For a given RB, denote the received SIR of a femto UE as SIR $_{F}$, the distance from an interfering FAP to the femto UE as $\left|X_{\mathrm{Fi}}\right|$, the path loss exponent on the link from the home FAP to the femto UE as $\alpha_{F}$, the path loss exponent on the link from an interfering FAP to the femto UE as $\alpha_{F F}$, the exponentially distributed channel power between interfering femtocell $\mathrm{FAP}_{0}$ and user 0 as $H_{0}$, the exponentially distributed channel power between interfering femtocell $\mathrm{FAP}_{i}$ and user 0 as $H_{0 i}$, the indoor lognormal shadowing experienced by user 0 as $\Theta_{0}$ and the lognormal shadowing experienced by user FAP as $\Theta_{0 i}$. Here, it is assumed that both $\Theta_{0}$ and $\Theta_{0 i}$ are independent and lognormally distributed. Let the mean and the standard deviation of the logarithmic distribution of $\Theta_{0}$ be $\mu$ and $\sigma$, respectively, and that of $\Theta_{0 i}$ be $\mu_{i}$ and $\sigma_{i}$, respectively, that is $\Theta_{0} \sim \operatorname{LN}\left(\zeta \mu, \zeta^{2} \sigma^{2}\right)$ and $\Theta_{0 i} \sim \operatorname{LN}\left(\zeta \mu_{i}, \zeta^{2} \sigma_{i}^{2}\right)$, where $\zeta \equiv 0.1 \ln 10$ is a scaling constant. Denote the index set corresponding to the lognormal shadowing experienced by user FAP as $\Phi$. $\phi$ is the wall penetration loss. Then we have

$$
\operatorname{SIR}_{\mathrm{F}}=\frac{\Theta_{0} H_{0} r_{\mathrm{F}}^{-\alpha_{\mathrm{F}}}}{\sum_{i \in \Phi} \Theta_{0 i} \phi^{2} H_{0 i}\left|X_{\mathrm{Fi}}\right|^{-\alpha_{\mathrm{FF}}}} .
$$

For the worst case scenario, it is assumed that all femto UEs are located on the edge of their home femtocells and there are wall penetration losses for links from an interfering FAP to the femto UE of interest.

The product of the shadowing and fading appearing in the numerator and denominator of (3), leads to a composite lognormal exponential distribution that is assumed to obey the following assumption:

i) The distribution of a composite lognormal exponential is modeled as lognormal distribution using Turkmani's approximation [19].

Using the above assumptions, $\Theta_{0}$ and $H_{0}$ are approximated by a lognormal random variable denoted as $\psi_{0}$. Similarly, $\Theta_{i}$ and $H_{i}$ are approximated by a lognormal random variable denoted as $\psi_{0 i}$. Then, (3) can be simplified as

$$
\operatorname{SIR}_{\mathrm{F}}=\frac{\psi_{0} r_{\mathrm{F}}^{-\alpha_{\mathrm{F}}}}{\sum_{i \in \Phi} \psi_{0 i} \phi^{2}\left|X_{\mathrm{Fi}}\right|^{-\alpha_{\mathrm{FF}}}} \cdot
$$

Allowing the cumulative distribution function (CDF) of the SIR of the femtocell to be calculated as 


$$
\begin{aligned}
& \operatorname{Pr}\left(\operatorname{SIR}_{\mathrm{F}} \leq \Gamma\right)=\operatorname{Pr}\left(\frac{\psi_{0} r_{\mathrm{F}}^{-\alpha_{\mathrm{F}}}}{\sum_{i \in \Phi} \psi_{0 i} \phi^{2}\left|X_{\mathrm{Fi}}\right|^{-\alpha_{\mathrm{FF}}}} \leq \Gamma\right) \\
& =\operatorname{Pr}\left(\sum_{i \in \Phi} \psi_{0 i} \phi^{2}\left|X_{\mathrm{Fi}}\right|^{-\alpha_{\mathrm{FF}}} \geq \frac{\psi_{0} r_{\mathrm{F}}^{-\alpha_{\mathrm{F}}}}{\Gamma}\right) \\
& \geq 1-E_{\psi_{0}}\left(\exp \left(-\pi \lambda_{f} \rho_{F} E\left(\psi_{I}^{\delta_{f}}\right)\left(\frac{\Gamma}{\psi_{0} r_{\mathrm{F}}^{-\alpha_{\mathrm{F}}}}\right)^{\delta_{f}}\right)\right) \\
& =1-E_{\psi_{0}}\left(\exp \left(-\rho_{\mathrm{F}} k_{f} \Gamma^{\delta_{f}} \psi_{0}^{-\delta_{f}}\right)\right),
\end{aligned}
$$

where $\delta_{f} \equiv \frac{2}{\alpha_{\mathrm{FF}}}, \psi_{I}$ is the equivalent value of $\psi_{i} \forall i \in \Phi$, $k_{f} \equiv \pi \lambda_{f} E\left(\psi_{I}^{\delta_{f}}\right) r_{\mathrm{F}}^{\alpha_{\mathrm{F}} \delta_{f}}, E_{\psi_{0}}(\cdot)$ and $E\left(\psi_{I}^{\delta_{f}}\right)$ are the expected value with respect to $\psi_{0}$ and $\psi_{I}$, respectively. Hence, the expected throughput of femtocell is

$$
\begin{aligned}
& T_{f}=\sum_{l=1}^{L-1} l \operatorname{Pr}\left(\Gamma_{l} \leq \operatorname{SIR}_{\mathrm{F}} \leq \Gamma_{l+1}\right)+L \operatorname{Pr}\left(\operatorname{SIR}_{\mathrm{F}} \geq \Gamma_{l}\right) \\
& =\sum_{l=1}^{L-1} l E_{\psi_{0}}\left(\exp \left(-\rho_{F} k_{f} \Gamma_{l}^{\delta_{f}} \psi_{0}^{-\delta_{f}}\right)-\exp \left(-\rho_{F} k_{f} \Gamma_{l+1}^{\delta_{f}} \psi_{0}^{-\delta_{f}}\right)\right) \\
& +L E_{\psi_{0}}\left(\exp \left(-\rho_{F} k_{f} \Gamma_{L}^{\delta_{f}} \psi_{0}^{-\delta_{f}}\right)\right)
\end{aligned}
$$

and the ASE is

$$
\begin{aligned}
& \operatorname{ASE}_{F}\left(\rho_{F}\right)=\rho_{F} \lambda_{f} T_{f} \\
& =\rho_{F} \lambda_{f}\left(\begin{array}{l}
\left.\sum_{l=1}^{L-1} l E_{\psi_{0}}\left(\exp \left(-\rho_{F} k_{f} \Gamma_{l}^{\delta_{f}} \psi_{0}^{-\delta_{f}}\right)-\exp \left(-\rho_{F} k_{f} \Gamma_{l+1}^{\delta_{f}} \psi_{0}^{-\delta_{f}}\right)\right)\right) \cdot(7) \\
+L E_{\psi_{0}}\left(\exp \left(-\rho_{F} k_{f} \Gamma_{L}^{\delta_{f}} \psi_{0}^{-\delta_{f}}\right)\right)
\end{array}\right)
\end{aligned}
$$

Hence, the decentralized spectrum resource allocation problem can be formulated as the following optimization problem:

\section{Problem ( $\left.\mathbf{P}^{\prime}\right)$}

$$
\begin{array}{ll}
\max _{\rho_{F}} & \operatorname{ASE}_{F}\left(\rho_{F}\right), \\
\text { subject to } & 0<\rho_{F} \leq 1 .
\end{array}
$$

This maximization problem is further equivalent to the following minimization problem:

\section{Problem (P)}

$$
\begin{array}{ll}
\min _{\rho_{F}} & -\operatorname{ASE}_{F}\left(\rho_{F}\right), \\
\text { subject to } & 0<\rho_{F} \leq 1 .
\end{array}
$$

\section{SOLUTION METHOD}

The optimal decentralized resource allocation problem defined in Problem (P) is actually a nonconvex optimization problem. In this paper, a filled function method [20]-[25] is employed for solving the global minimum of Problem (P).

A filled function [20]-[25] is a function for which (1) the current local minimum of the original cost function is the current local maximum of the filled function; (2) the whole current basin of the original cost function is a part of the current hill of the filled function; (3) the filled function has no stationary point in any higher basins of the original cost function; and (4) there exists a local minimum of the filled function which is in a lower basin of the original cost function.
Some terminologies related to filled functions have been used above. Notably, a basin of a function is defined as the subset of the domain of the optimization variables such that any points in this subset will give the same local minimum of the function via conventional gradient based optimization methods. Furthermore, a hill of a function is defined as the subset of the domain of the optimization variables such that any points in this subset will give the same local maximum of the function via conventional gradient based optimization methods. Also, a higher basin of a function is a basin of the function with the cost value of the local minimum of the basin is higher than that of the current basin of the function. Finally, a lower basin of a function is a basin of the function with the cost value of the local minimum of the basin is lower than that of the current basin of the function.

Due to property 1), by evaluating the filled function at a point slightly deviated from the current local minimum of the original cost function; a lower filled function value is obtained. Hence, the filled function could kick away from the current local minimum of the original cost function. Due to properties (2)-(4), the current local minimum of the filled function is neither in the current basin nor any higher basins of the original cost function. Hence, the current local minimum of the filled function is in a lower basin of the original cost function. As a result, by solving the next local minimum of the originally cost function searching at the neighborhood around the current local minimum of the filled function, a better local minimum of the original cost function is obtained. If the original cost function contains finite number of local minima, then the global minimum of the original cost function will be eventually reached.

The following algorithm is proposed for solving the global minimum of Problem (P):

Algorithm

Step 1:Initialize a minimum improvement factor $\varepsilon$, an accepted error $\varepsilon^{\prime}$, an initial search point $\tilde{\rho}_{F, 1}$, a positive number $R$ and an iteration index $n=1$.

Step 2:Solve a local minimum of the following optimization Problem $\left(\mathbf{P}_{f}\right)$ using a conventional gradient based approach with the initial search point $\tilde{\rho}_{F, n}$.

\section{$\operatorname{Problem}\left(\mathbf{P}_{f}\right)$}

$$
\begin{array}{ll}
\min _{\rho_{F}} & (9 \mathrm{a}), \\
\text { subject to } & (9 \mathrm{~b}) \\
\text { and } & \operatorname{ASE}_{F}\left(\tilde{\rho}_{F, n}\right)-(1-\varepsilon) \operatorname{ASE}_{F}\left(\rho_{F}\right) \leq 0,
\end{array}
$$

where $\operatorname{ASE}_{F}\left(\tilde{\rho}_{F, n}\right)-(1-\varepsilon) \operatorname{ASE}_{F}\left(\rho_{F}\right) \leq 0$ is the constraint function we imposed. Denote the obtained local minimum as $\rho_{F, n}^{*}$.

Step 3:Solve a local minimum of the following optimization Problem $\left(\mathbf{P}_{H}\right)$ using a conventional gradient based approach with the initial search point $\rho_{F, n}^{*}$.

\section{Problem $\left(\mathbf{P}_{H}\right)$}

$$
\min _{\rho_{F}} \quad H\left(\rho_{F}\right) \equiv-\operatorname{ASE}_{F}\left(\rho_{F}\right)+\frac{1}{\left(\rho_{F}-\rho_{F, n}^{*}\right)^{T} R\left(\rho_{F}-\rho_{F, n}^{*}\right)},
$$


subject to (9b)

$$
\text { and } \quad \operatorname{ASE}_{F}\left(\rho_{F, n}^{*}\right)-(1-\varepsilon) \operatorname{ASE}_{F}\left(\rho_{F}\right) \leq 0 \text {, }
$$

where $H\left(\rho_{F}\right)$ is the filled function we define and $\operatorname{ASE}_{F}\left(\rho_{F, n}^{*}\right)-(1-\varepsilon) \operatorname{ASE}_{F}\left(\rho_{F}\right) \leq 0$ is the constraint function we imposed. Denote the obtained local minimum as $\tilde{\rho}_{F, n+1}$. Increment the value of $n$.

Step 4:Iterate Step 2 and Step 3 until $\operatorname{ASE}_{F}\left(\rho_{F, n}^{*}\right)-\operatorname{ASE}_{F}\left(\rho_{F, n-1}^{*}\right) \leq \varepsilon^{\prime}$. Take the final value of $\rho_{F, n}^{*}$ as the global minimum of Problem (P).

Step 1 is an initialization of the proposed algorithm. In order not to terminate the algorithm when the convergence of the algorithm is slow and to have a high accuracy of the solution, both $\varepsilon$ and $\varepsilon^{\prime}$ should be chosen as small values. Also, as $\tilde{\rho}_{F, 1}$ is an initial search point of the optimization algorithm, this initial search point should be in the feasible set. Moreover, as $R$ is a positive number, it controls the spread of the hill of $H\left(\rho_{F}\right)$ at $\rho_{F, n}^{*}$. A large value of $R$ will result in a wide spread of the hill of $H\left(\rho_{F}\right)$ at $\rho_{F, n}^{*}$ and vice versa. Since the local minima of nonconvex optimization problems could be located very close together, the spread of the hill of $H\left(\rho_{F}\right)$ at $\rho_{F, n}^{*}$ should be small and $R$ should be chosen as a small positive number. Step 2 is to find a local minimum of $-\operatorname{ASE}_{F}\left(\rho_{F}\right)$. As the constraint (9c) is imposed on the Problem $\left(\mathbf{P}_{f}\right)$, the obtained ASE is guaranteed to be larger than that corresponding to $\tilde{\rho}_{F, n}$. Similarly, Step 3 is to find a local minimum of $H\left(\rho_{F}\right)$. As the constraint (10b) is imposed on the Problem $\left(\mathbf{P}_{H}\right)$, the obtained ASE is guaranteed to be larger than that corresponding to $\rho_{F, n}^{*}$. Step 4 is a termination test procedure. If the difference of the ASEs between two consecutive iterations is smaller than a certain bound $\varepsilon^{\prime}$, then the algorithm is terminated.

It has been discussed before that the filled function method has to satisfy four properties. As $R$ is a positive number and $\rho_{F, n}^{*}$ is in the denominator of $H\left(\rho_{F}\right)$, $H\left(\rho_{F}\right) \rightarrow+\infty$ as $\rho_{F} \rightarrow \rho_{F, n}^{*}$. Hence, $\rho_{F, n}^{*}$ is the global maximum of $H\left(\rho_{F}\right)$ and property (1) is guaranteed to be satisfied. As the constraint (10b) is imposed on the Problem $\left(\mathbf{P}_{H}\right)$, when a new local minimum of $H\left(\rho_{F}\right)$ is found, this new local minimum of $H\left(\rho_{F}\right)$ will not be located at $\rho_{F, n}^{*}$ and the ASE evaluated at $\tilde{\rho}_{F, n+1}$ will guarantee to be larger than that at $\rho_{F, n}^{*}$. Hence, properties (2)-(4) are guaranteed to be satisfied. As a result, the proposed algorithm guarantees to reach the global minimum of Problem (P).

Compared to existing works in which all possible values of the fraction of radio resources per transmission interval accessible by each femtocell are evaluated [12], our proposed method only evaluates the stationary points of the cost function. As the total number of evaluation points is reduced significantly, the computational effort for solving the globally optimal solution of the optimization problem is significantly reduced.

\section{COMPUTER NUMERICAL SIMULATIONS}

In order to have a fair comparison between our proposed method and the scheme in [12], the same sets of parameter values used in [12] are employed in our simulations. These include, $L=8, \delta_{f}=0.5714$, $\Gamma_{l}=\left(2^{l}-1\right) \times 10^{0.3}$ for $l=1,2, \cdots, 8, \mu=0 \mathrm{~dB}, \sigma^{2}=4 \mathrm{~dB}$, $\mu_{i}=0 \mathrm{~dB} \forall i \in \Phi, \sigma_{i}^{2}=12 \mathrm{~dB} \forall i \in \Phi, \lambda_{f}=4.6405 \times 10^{-5}$ and $k_{f}=0.0813 \times E\left(\psi_{I}^{\delta_{f}}\right)$ for $N_{f}=10, \lambda_{f}=2.3202 \times 10^{-4}$ and $k_{f}=0.4065 \times E\left(\psi_{I}^{\delta_{f}}\right)$ for $N_{f}=50$, and $\lambda_{f}=4.6405 \times 10^{-4}$ and $k_{f}=0.813 \times E\left(\psi_{I}^{\delta_{f}}\right)$ for $N_{f}=100$. Besides, for the proposed filled function method, $\varepsilon=\varepsilon^{\prime}=10^{-6}$ and $R=10^{-3}$ are chosen because they are small enough for most applications, and $\tilde{\rho}_{F, 1}=0.5$ is chosen because it is the midpoint in the feasible region.

In [12], the tested range of $\rho_{F}$ is from $\rho_{F}=0.01$ to $\rho_{F}=1$, and a step size of 0.01 is used. Accordingly, there are a hundred points to be evaluated in this test range. By using our proposed filled-function-based algorithm, we obtain the same set of optimal values of $\rho_{F}$ as those obtained in [12]. That is, $\rho_{F}=1$ for $N_{f}=10, \rho_{F}=0.55$ for $N_{f}=50$ and $\rho_{F}=0.3$ for $N_{f}=100$. Moreover, it takes only one iteration for our proposed method to reach the globally optimal solution for the above values of $N_{f}$. Therefore, our proposed method is more efficient than the one discussed in [12].

\section{CONCLUSIONS}

In this paper, a filled function method is employed for deriving the globally optimal decentralized resource allocation scheme for OFDMA downlink of a coexisting macro/femto network. The ASE is maximized subject to a constraint on the radio resources per transmission interval accessible by each femtocell. As the proposed method only evaluates the stationary points of the cost function, the computational effort for solving the globally optimal solution of the optimization problem is significantly reduced.

\section{REFERENCES}

[1] Shu-ping Yeh, Shilpa Talwar, Seong-Choon Lee and Heechang Kim, "WiMAX femtocells: a perspective on network architecture, capacity, and coverage,” IEEE Communications Magazine, vol. 46, no. 10, pp. 58-65, 2008.

[2] 3GPP LTE, http://www.3gpp.org/Highlights/LTE/LTE.htm.

[3] IEEE 802.16m-07/002r4, “TGm System Requirements Document (SRD),” approved 19 Oct 2007.

[4] Vikram Chandrasekhar, Jeffrey G. Andrews and Alan Gatherer, "Femtocell networks: a survey," IEEE Communications Magazine, vol. 46, no. 9, pp. 59-67, 2008.

[5] Malcolm Latham, "Consumer attitudes to femtocell enabled inhome services -insights from a European survey," Femtocells Europe, 23-25 June, 2008.

[6] Alexander L. Stolyar and Harish Viswanathan, "Self-organizing dynamic fractional frequency reuse in OFDMA systems," The $27^{\text {th }}$ IEEE Conference on Computer Communications, pp. 691-699, 1318 April, 2008.

[7] S-E. Elayoubi, O. Ben Haddada and B. Fourestié, "Performance evaluation of frequency planning schemes in OFDMA-based networks," IEEE Transactions on Wireless Communications, vol. 7, no. 5, pp.1623-1633, 2008. 
[8] M. Assaad and A. Mourad, "New frequency-time scheduling algorithms for 3GPP/LTE-like OFDMA air interface in the downlink,” IEEE Vehicular Technology Conference, VTC Spring, pp. 1964-1969, 11-14 May, 2008.

[9] Marc C. Necker, "Local interference coordination in cellular OFDMA networks," IEEE $66^{\text {th }}$ Vehicular Technology Conference, VTC Fall, pp. 1741-1746, 30 September - 3 October, 2007.

[10] Arne Simonsson, "Frequency reuse and intercell interference coordination in E-UTRA," IEEE $65^{\text {th }}$ Vehicular Technology Conference, VTC Spring, pp. 3091-3095, 22-25 April, 2007.

[11] David López-Pérez, Guillaume de la Roche, Alvaro Valcarce, Alpár Jüttner and Jie Zhang, "Interference avoidance and dynamic frequency planning for WiMAX femtocells networks,” $11^{\text {th }}$ IEEE Singapore International Conference on Communication Systems, ICCS, pp. 1579-1584, 19-21 November, 2008.

[12] Vikram Chandrasekhar and Jeffrey G. Andrews, "Spectrum allocation in tiered cellular networks," IEEE Transactions on Communications, vol. 57, no. 10, pp. 3059-3068, 2009.

[13] Vikram Chandrasekhar and Jeffrey G. Andrews, "Spectrum allocation in two-tier networks," $42^{\text {th }}$ Asilomar Conference on Signals, Systems and Computers, pp. 1583-1587, 26-29 October, 2008.

[14] Vikram Chandrasekhar, Marios Kountouris and Jeffreys G. Andrews, "Coverage in multi-antenna two-tier networks," IEEE Transactions on Wireless Communications, vol. 8, no. 10, pp. 5314-5327, 2009.

[15] 3GPP Release 8, http://www.3gpp.org/Release-8.

[16] Steven Weber, Jeffrey G. Andrews and Nihar Jindal, "The effect of fading, channel inversion, and threshold scheduling on ad hoc networks," IEEE Transactions on Information Theory, vol. 53, no. 11, pp. 4127-4149, 2007.

[17] Vikram Chandrasekhar, Jeffrey G. Andrews, Tarik Muharemovic, Zukang Shen and Alan Gatherer, "Power control in two-tier femtocell networks,” IEEE Transactions on Wireless Communications, vol. 8, no. 8, pp. 4316-4328, 2009.

[18] Jeffrey Andrews, Arunabha Ghosh and Rias Muhamed, Fundamentals of WiMAX: Understanding Broadband Wireless Networking, Prentice Hall, 2007.

[19] A. M. D. Turkmani, "Probability of error for M-branch macroscopic selection diversity," IEE Proceedings I:-Communications, Speech and Vision, vol. 139, no. 1, pp. 7178, 1992.

[20] Ying Zhang, Liansheng and Yingtao Xu, "New filled functions for nonsmooth global optimization,” Applied Mathematical Modelling, vol. 33, pp. 3114-3129, 2009.

[21] Xian Liu, "Finding global minima with a computable filled function,” Journal of Global Optimization, vol. 19, pp. 151-161, 2001.

[22] K. F. C. Yiu, Y. Liu and K. L. Teo, "A hybrid descent method for global optimization,” Journal of Global Optimization, vol. 28, pp. 229-238, 2004.

[23] Z. Y. Wu, H. W. J. Lee, L. S. Zhang and X. M. Yang, “A novel filled function method and quasi-filled function method for global optimization," Computational Optimization and Applications, vol. 34, pp. 249-272, 2005.

[24] R. P. Ge and Y. F. Qin, “A class of filled functions for finding global minimizers of a function of several variables," Journal of Optimization Theory and Applications, vol. 54, no. 2, pp. 241-252, 1987.

[25] Renpu Ge, "A filled function method for finding a global minimizer of a function of several variables," Mathematical Programming, vol. 46, pp. 191-204, 1990. 